ÉGYPTE

monde arabe

\section{Égypte/Monde arabe}

15-16 | 1993

Les crises soudanaises des années 80

\title{
Les crises soudanaises des années 80 : Présentation
}

Édouard Conte et François Ireton

\section{Q OpenEdition}

\section{Journals}

Édition électronique

URL : https://journals.openedition.org/ema/1004

DOI : 10.4000/ema.1004

ISSN : 2090-7273

Éditeur

CEDEJ - Centre d'études et de documentation économiques juridiques et sociales

Édition imprimée

Date de publication : 31 décembre 1993

Pagination : 7-20

ISSN : 1110-5097

\section{Référence électronique}

Édouard Conte et François Ireton, «Les crises soudanaises des années 80 : Présentation », Égypte/ Monde arabe [En ligne], 15-16 | 1993, mis en ligne le 08 juillet 2008, consulté le 07 juillet 2022. URL: http://journals.openedition.org/ema/1004; DOI : https://doi.org/10.4000/ema.1004

Ce document a été généré automatiquement le 7 juillet 2022.

Tous droits réservés 


\title{
Les crises soudanaises des années 80 : Présentation
}

\author{
Édouard Conte et François Ireton
}

\section{NOTE DE L'ÉDITEUR}

Ce numéro d'Égypte/Monde arabe n'aurait pu paraître sans l'accueil chaleureux et patient des professeurs Salah al-Din al-Shazali et Mustala Babiker Ahmed, tous deux du Development Studies and Research Centre (DSRC) de l'Université de Khartoum, qui ont manifesté leur désir d'établir des liens scientifiques et amicaux avec des chercheurs français et ont accordé aux responsables de cette livraison leur aide et celle de leur institution. Mustafa Kharoufi, dans un premier temps et Rupert Hasterok dans un second ont assuré avec efficacité les liaisons, institutionnellement aisées mais matériellement difficiles, entre le DSRC et le CEDEJ.

Il faut enfin mentionner tout spécialement le travail minutieux de préparation à l'édition qu'ont effectué Édouard Conte et François Ireton, co-éditeurs de ces numéros, qui ont notamment assumé la révision et l'harmonisation des textes originaux puis avec la collaboration de Samia Rizq, traductrice, Sylvia Chiffoleau, Iman Farag et Alain Roussillon, chercheurs au CEDEJ - l'essentiel de la traduction.

Que toutes et tous soient ici chaleureusement remerciés.

1 Ce dossier est constitué, dans sa quasi-totalité, de contributions de chercheurs et universitaires soudanais proposant une analyse de certains des problèmes cruciaux que leur pays a connus depuis le début des années 80. Les articles qui le composent correspondent ${ }^{1}$ aux communications qui devaient initialement être présentées lors d'un colloque portant sur le même thème, prévu à Paris pour décembre 1991 et qui n'a pu avoir lieu du fait de l'impossibilité où se trouvaient certains de ses participants soudanais de quitter leur pays. Ce colloque était organisé conjointement par le DSRC (Development Studies and Research Center) de l'Université de Khartoum et le CEDEJ (Centre d'études et de documentation économique, juridique et sociale) du Caire, à l'initiative de Salah al-Din al-Shazali, enseignant-chercheur au DSRC et d'Édouard 
Conte, ancien chercheur au CEDEJ, chargé de recherches au CNRS et membre du Laboratoire d'anthropologie sociale (LAS) du Collège de France. L'initiative, soutenue par la direction du DSRC, avait été efficacement - et matériellement - encouragée par Jean-Claude Vatin, directeur de recherches au CNRS, alors directeur du CEDEJ, et facilitée par Françoise Héritier-Auger, professeur au Collège de France, directrice du LAS ainsi que par André Miquel, également professeur au Collège de France et administrateur général de cette institution où, grâce à sa bienveillance et son intérêt, le colloque devait avoir lieu. Les circonstances en ont décidé autrement, témoignant des difficultés que connaissent les chercheurs soudanais tant à poursuivre leurs travaux qu'à les faire connaître, et ceci pour des raisons tout à la fois politiques et économiques, en partie liées aux crises qui faisaient l'objet de la réunion prévue.

2 Si bien des colloques voient la publication de leurs actes (parfois indéfiniment) différée, il est rare que soient publiés des actes de colloques qui n'ont pas eu lieu... On trouvera pourtant ici réalisé ce cas de figure inédit, non par goût du paradoxe mais pour répondre à deux objectifs.

3 Le premier réside dans l'analyse de la réalité soudanaise, qui vient au second rang des préoccupations de la revue Égypte/Monde arabe après celle du devenir de l'Égypte. Les publications périodiques du CEDEJ avaient déjà antérieurement (depuis 1986) abordé l'actualité et la réalité socio-historique soudanaises². Depuis le premier numéro de la revue, une place particulière a été ménagée à l'actualité soudanaise, en général si ignorée - et caricaturée - par les médias français ${ }^{3}$, dans la chronologie politique. Ce numéro double continue donc une "tradition", trop sporadiquement respectée jusqu'ici, mais qui devrait l'être plus régulièrement dorénavant, d'autant qu'un pôle de recherches sur le Soudan (en particulier sur la région de Kassala, située à l'est du pays) se met en place au CEDEJ, en collaboration avec certains des chercheurs soudanais contributeurs à ce numéro. Pour preuve de ces bonnes résolutions, celui-ci sera donc immédiatement suivi d'un autre ( $\mathrm{n}^{\circ} 17,1 \mathrm{er}$ trimestre 1994), qui rassemblera des articles de chercheurs en majorité français, appartenant au CEDEJ ou coopérant avec ce dernier et désireux de prendre part, selon leur spécialité, à la constitution d'un tableau du Soudan à l'orée des années $90^{4}$. Il eût été certes souhaitable de mêler recherches soudanaises et françaises (ou autres) sur ce pays mais, outre le volume trop important - même pour un numéro double - qu'aurait représenté cet ensemble, priorité a été donnée à la publication des contributions au colloque de 1991.

Notre deuxième objectif - commun à la rencontre et à cette publication - est en effet de faire connaître, en langue française, à un public moins étroit que celui des seuls spécialistes français du Soudan, qui ne sont guère plus d'une douzaine ${ }^{5}$, des recherches en sciences sociales menées par des chercheurs soudanais sur leur propre pays. Dix de ces derniers avaient manifesté le désir de participer au colloque de Paris et non seulement d'y exposer une recherche "pointue ", mais d'y parler librement de leur travaux ainsi que des problèmes et de l'évolution de la recherche au Soudan avec les nombreux chercheurs français, africanistes et spécialistes des pays arabes et musulmans qui avaient fait connaître leur volonté de participer à cette rencontre. Il est à souhaiter que ce numéro - et le suivant - d'Égypte/Monde arabe, à défaut de permettre l'échange direct, réponde en partie à cet objectif de contribution à une meilleure "visibilité » du Soudan et des recherches soudanaises sur ce pays, dans le champ francophone des sciences sociales. 
5 «Les crises soudanaises dans les années 80 » : ce sont les participants soudanais au colloque qui ont eux-mêmes proposé ce titre. Il s'agissait d'analyser sous le nom de " crise » - terme dont on a sans doute abusé mais qui, en l'espèce, parait ici tout à fait adapté - les graves difficultés que le Soudan a connues depuis ce que l'on pourrait appeler le «retournement de conjoncture globale» qui s'est produit à l'orée de la dernière décennie. C'est à cette époque, en effet, qu'un certain nombre d'indicateurs se sont « mis au rouge " dans ce pays, à commencer par ceux synthétisant son évolution socio-économique. Dans ce domaine, le Soudan qui n'évoque plus aujourd'hui pour l'opinion publique - quand bien même il évoque quelque chose - que sécheresse, famine, endettement, pauvreté et déplacements de population, avait indiscutablement connu durant les années 70 une phase de croissance - si ce n'est de développement économique.

6 Dans «Sous-développement, crise globale et crise économique au Soudan », Salah alDin al-Shazali analyse les racines coloniales et post-coloniales de cette crise qu'il refuse d'envisager comme une collection de crises sectorielles indépendantes, mais considère comme une crise unique, à multiples facettes, dont tous les éléments, socioéconomiques, écologiques et politiques sont liés. C'est autour du développement d'une économie d'exportation de produits primaires agricoles et d'importation de produits manufacturés, ceci sous l'égide d'un capitalisme marchand peu enclin aux investissements productifs à long terme mais au contraire très mobile et cherchant les profits rapides, qu'il voit s'articuler les causes de cette crise globale. Medani M. Ahmed en décrit les grandes manifestations économiques dans "La crise de l'économie soudanaise dans les années 80 " et revient sur l'absence d'une bourgeoisie entrepreneuriale au Soudan et d'un réel « moteur industriel »; il insiste également sur l'endettement. Ces deux articles forment l'introduction.

7 C'est sur le thème complexe des aspects écologique, agricole et alimentaire de la crise globale qui a caractérisé les années 80 et de leurs conséquences, que portent les six articles qui forment la première partie. Mustafa Babiker envisage, dans «Critique de l'économie politique de l'insécurité alimentaire; réflexions à partir de l'expérience soudanaise ", les différents cadres explicatifs utilisés pour l'analyse des crises de subsistance; si, d'un côté, il critique sans réserve les explications par les déterminations naturelles et l'«arriération», il montre, de l'autre, les limites des explications "dépendantistes" faisant uniquement appel à l'impérialisme, à l'intégration au marché mondial et à la pénétration du capitalisme. Non pas que ces derniers phénomènes - comme d'ailleurs la fragilité de l'écosystème sahélien - ne constituent pas des réalités à prendre impérativement en compte, mais en privilégiant ainsi des formes de "déterminisme externe", les tenants de ce type d'explication négligent l'analyse des structures sociales et cognitives paysannes, ainsi que la complexité des rapports sociaux «internes» qui lient les nombreux groupes différenciés d'agents de la production et de la commercialisation des denrées agricoles.

Un bon exemple d'analyse de telles structures complexes est livré, à propos de la grande agriculture pluviale mécanisée, par l'article d'Abdelgalil Elmekki sur "L'agriculture mécanisée : crise de production, crise de reproduction». C'est sur ce secteur agricole que devait s'appuyer, on le sait, la mise en œuvre de la stratégie du «breadbasket par laquelle le Soudan, censément riche d'un énorme potentiel de terres arables, non content - ce qui était primordial - d'assurer sa propre sécurité alimentaire, devait devenir le «grenier à blé » d'un Moyen-Orient dont les conditions 
naturelles difficiles - jointes à des politiques erronées ayant conduit à négliger le développement des secteurs agricoles nationaux - ont fait l'une des régions les plus déficitaires du globe sur le plan alimentaire. C'est à l'analyse des raisons de cette double incapacité à assurer une sécurité alimentaire tant intérieure que moyenorientale que se livre l'auteur, raisons qu'il découvre dans les caractéristiques propres au secteur mécanisé : structures économiques internes et dispositifs juridiques qui favorisent, là encore, l'obtention de profits rapides et spéculatifs au détriment de la reproduction de fertilité de sols vite épuisés, fonctions sociopolitiques et mode particulier de rapport à l'État, bailleur des terres et grand pourvoyeur de subventions à capter et qui, en retour, s'assure une clientèle par le biais de l'attribution avantageuse des grandes exploitations. Le secteur mécanisé, loin de ne connaître qu'une crise de production - une incapacité passagère à atteindre les objectifs quantitatifs qui lui sont fixés - subit une crise de reproduction, incapable qu'il est d'assurer la pérennisation des conditions naturelles et économiques de son fonctionnement et le niveau de revenu escompté de ceux qui visaient à en être les bénéficiaires et qui, pratiquant la fuite en avant, mettent toujours plus en péril la viabilité écologique et économique de ce secteur. Les trois articles suivants de cette partie constituent des analyses régionales très fouillées de crises de subsistance et de la vulnérabilité qui en découle pour de larges fractions des populations touchées. De plus, ces analyses ont en commun le fait qu'elles n'isolent pas ces aspects de la crise globale de ses autres aspects, sociopolitiques en particulier, qu'il s'agisse des conflits d'intérêts qui opposent des groupes pastoraux semi-nomades et agricoles sédentaires très inégalement relayés et défendus par le pouvoir d'État et ses administration de gestion des ressources naturelles et agricoles, des dissidences et conflits armés régionaux, ou des contradictions nationales/internationales en matière de gestion et d'orientation de l'aide, des premiers secours à l'assistance aux projets de développement et de restauration du milieu naturel. L'article de Catherine Miller, intitulé «Famine, conflits et aide internationale au Darfour (1990-1992) " s'appuie sur une riche expérience de terrain acquise à l'occasion de missions effectuées au Darfour dans le cadre de l'UNDP et décrit de manière détaillée et articulée entre elles à la fois les conséquences de la sécheresse et des famines ayant sévi cruellement dans cette région depuis maintenant dix ans, les stratégies d'adaptation des populations à la dégradation de leur mode de vie, l'action de l'État et des organismes humanitaires et l'intrication de ces phénomènes avec les différentes formes de dissidence liées tant au banditisme qu'aux conflits régionaux, interethniques et nord-sud soudanais dont le Darfour est le théâtre.

Reposant aussi sur une grande familiarité avec le terrain, liée à une expérience concrète de chercheur consultant, l'article de Salah al-Din al-Shazali, «Famine/ vulnérabilité et politique étatique: le cas des éleveurs du sud de la province de Kassala ", vise à la même mise en rapport - en y introduisant de plus la dimension historique de la longue durée - des déterminants de la situation précaire actuelle des pasteurs de cette région. Identités ethnico-tribales, zones naturelles complémentaires, implantation des points d'eau, logique des transhumances annuelles, -fixation des territoires des différents groupes, organisation de la gestion des ressources naturelles, articulation des pouvoirs locaux, régionaux et étatique, tout cela avait été enserré dans un ensemble de règlements attentifs à préserver la complexité des équilibres tant écologiques que socio-économiques et, durant les quarante premières années de la période coloniale, "les activités pastorales dans la région se révélèrent florissantes ", écrit l'auteur. La seconde partie de l'article est consacrée à la description du processus 
de marginalisation progressive des pasteurs, qui a abouti à leur extrême vulnérabilité face à la sécheresse, ceci essentiellement sous l'impact de l'implantation, dans la région, des grandes exploitations d'agriculture pluviale mécanisée et des conséquences de cette expansion. C'est dans la province du Nil Blanc, entre le Nord-Kordofan et le bras du Nil qui donne son nom à cette province, que s'est déroulée l'enquête dont rend compte l'avant-dernier article de cette partie, consacré aux stratégies migratoires déployées par des groupes domestiques paysans face à la sécheresse de 1983-1985. L'auteur, Ahmad Taha Mohammed, dans «Les mouvements de population provoqués par la sécheresse : une approche microsociologique ", caractérise avec précision deux faisceaux de stratégies correspondant à deux catégories de migrants : ceux qui ont quitté « sans retour » leur village pour la « capitale » de la province et ceux qui, après un séjour plus ou moins long en ville, sont retournés dans leur village d'origine. Les premiers sont plus alphabétisés, ils avaient, avant même leur départ vers la ville, davantage de contacts avec elle et ont parfois anticipé la famine de 1984-85 en migrant en ville avant qu'elle ne survienne, dans le cadre de stratégies de mobilité à la fois spatiale et socioprofessionnelle; les seconds ont migré sous la pure «contrainte » des événements et ont gardé toutes les caractéristiques (en termes de mode de vie, de consommation, de contact avec la culture écrite, etc.) de la petite paysannerie « traditionnelle » pour laquelle la ville ne pouvait en aucun cas constituer un milieu de vie définitif.

La vulnérabilité, socialement produite, face aux désastres dont les caractères «naturels » eux-mêmes sont l'objet d'un constant examen critique dans les articles précédents, ne concerne pas que les populations rurales, agricoles et/ou pastorales; les résidants urbains des quartiers populaires y sont aussi confrontés, comme ce fut le cas à Khartoum lors des terribles pluies qui s'abattirent sur la ville durant le mois d'août 1988. Dans un article tiré d'un rapport d'enquête menée sur le vif pour une grande ONG - OXFAM America - et intitulé «Les inondations de Khartoum en 1988: impact et réaction des populations" (article figurant dans la même partie que les précédents dans la mesure où il concerne aussi une situation de crise engendrée par des facteurs «naturels »), Mustafa Babiker décrit la réalité et les limites de la «résistance » et de l'auto-organisation des habitants face à la dévastation de leur quartier.

Le deuxième sous-ensemble de ce dossier est consacré aux aspects proprement politiques de la crise globale: le politique était bien évidemment déjà largement présent dans les articles de la première partie, essentiellement sous l'aspect de la gestion étatique des ressources naturelles et des liens privilégiés existant entre certaines catégories d'agents de la production agricole et l'État ou ses administrations. Symétriquement, le socio-économique est présent dans les deux articles de Salah al-Din al-Shazali qui ouvrent cette partie et qui traitent des conséquences, dans ce domaine, des conflits politiques hantant la société soudanaise : l'un sur le plan intérieur - la guerre civile du Sud-Soudan qui a repris depuis 1983 (« Le coût humain et social de la guerre civile ») - l'autre à sa périphérie : le conflit, maintenant clos, entre l'Ethiopie et son ex-province érythréenne, dont les réfugiés se sont ou ont été, pour la plupart, installés dans la région de Kassala, déjà citée («Les réfugiés étrangers»). Restant à l'interface des réalités socio-économiques et du pouvoir politique, l'article de Siddig Umbadda, «La «tribu» des diplômés et la mauvaise gestion de l'économie soudanaise ", explore le recrutement de la "technostructure " soudanaise et souligne l'existence des pratiques de « capture de rente » (rent-seeking) qui la caractérisent, ainsi que la surconcentration de hauts fonctionnaires originaires des régions centrales", 
deux phénomènes (le premier ayant déjà été aborde dans plusieurs articles de la première partie) qui expliqueraient la crise de gestion dont souffre également l'économe soudanaise.

L'histoire politique soudanaise s'est caractérisée, depuis l'indépendance, non seulement par ses conflits internes et externes, mais encore par l'alternance cyclique de pouvoirs civils et démocratiques et de pouvoirs militaires et autoritaires. Dans un article qui reprend sous un angle particulier cette histoire politique presque quarantenaire et qui s'intitule "Traditionalisme et modernisation: une perspective politique ", Mohammed Bechir Hamid défend la thèse selon laquelle une "démocratie parlementaire corrompue, quasi anarchique " aux mains des deux partis dominants à base confrérique et un "régime militaire de type conservateur et dictatorial " ne peuvent nullement être opposés comme la modernité à la tradition. Au contraire, l'auteur ne voit apparaître des "éléments modernistes » sur la scène du pouvoir d'État que lors des passages alternés d'un type de régime à l'autre, passage s'effectuant dans un contexte de crise politique que ces éléments contribuent à résoudre pour être ensuite bien vite écartés du pouvoir.

Le Front national islamique (FNI) issu pour une part du mouvement des Frères musulmans soudanais, apparu dans les années 40 , constitue-t-il un exemple de ces "éléments modernistes" favorisant le passage d'un type de régime à un autre? Présents dans le gouvernement démocratique issu des élections libres de 1986 qui se sont tenues un an jour pour jour après le renversement du régime militaire de Nimeyri, ils en ont démissionné quelques mois avant que les militaires, sous la conduite de Omar al-Bechir, ne reprennent le pouvoir en juin 1989, ceci dans un indiscutable contexte de crise politique liée à l'échec des tentatives de redressement de la situation économique et de conclusion d'accords de paix avec l'ALPS du Sud-Soudan. Le FNI fait l'objet de l'article d'Idris Salim, intitulé « Le Front national islamique, idéologie et pratique », qui en envisage moins les formes de participation au régime militaire actuel (participation dont les modalités réelles font l'objet des discussions des spécialistes), que ce que l'auteur appelle les mécanismes d'idéologisation » par lesquels le FNI a forgé et proposé son interprétation de la situation soudanaise et a mobilisé une fraction de la population autour de cette interprétation. Il était intéressant de confronter la vision et les pratiques islamistes à un "islam périphérique » soudanais tel qu'il est vécu par une ethnie située loin de la capitale, en l'occurrence ici dans le Darfour : c'est ce que permet de faire l'article sur "L'Islam chez les Berti » de Abdulahi Osman al-Tom. Les Berti constituent l'une des très nombreuses ethnies qui peuplent le Soudan. Ces dernières, loin de n'appartenir qu'à un héritage obsolète et figé, restent des réalités sans cesse réactualisées et mouvantes, qui jouent leur rôle dans et sont reproduites par les conflits et crises politiques "modernes»; c'est ce que montre - reprenant les théories du grand anthropologue norvégien Friedrik Barth (qui a enquêté dans le Darfour et formé des disciples soudanais) - Paul W. Gore qui fait également appel à son expérience pratique de médiateur dans certains conflits interethniques du Sud-Soudan.

14 Ce dossier s'achève avec le texte de Mohammed Ahmed Taisier : "Le développement comme outil de la paix : un rêve avorté ». Auteur d'un ouvrage très intéressant intitulé The cultivation of hunger (KUP, Khartoum, 1991) portant sur les politiques agricole et alimentaire des différents pouvoirs soudanais durant la période coloniale et les premières années de l'indépendance, $M$. A. Taisier, actuellement dans l'impossibilité de mener ses recherches universitaires, nous livre ici une brève analyse et des réflexions 
qui montrent que, quels que soient les échecs du développement - notion que l'auteur redéfinit - et le discrédit dans lequel cette notion est elle-même tombée dans certaines sphères, il existe des relations réciproques entre ce à quoi renvoie ce terme et l'état de paix auquel aspire l'immense majorité des Soudanais.

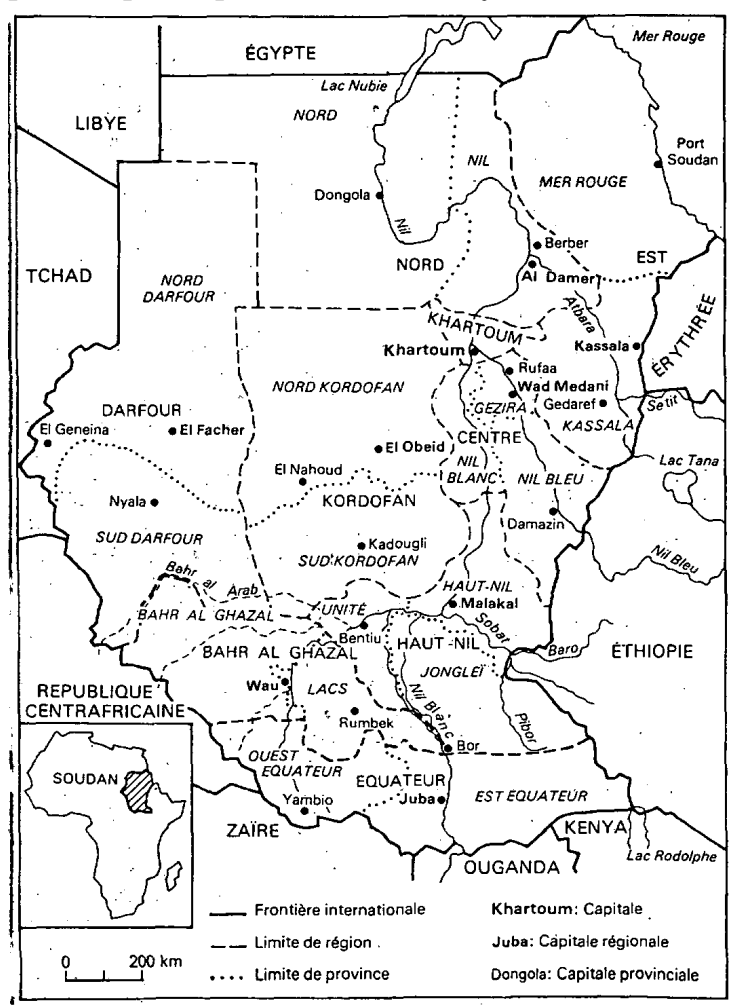

Carte générale du Soudan (divisions administratives) Source ; M. Lavergne et al. Le Soudan contemporain, Karthala-CERMOC, 1989, p. 4.

\section{NOTES}

1. Ces communications, rédigées à l'origine en anglais (sauf le texte de I. SALIM, dont l'original est en arabe) n'ont pu être remaniées par leurs auteurs que dans certains cas. Quelques traductions sont dites « adaptées »; cela signifie que, pour des raisons matérielles de place ou pour cause de redondance entre les articles, certains passages ont été résumés ou supprimés.

2. La Revue de la presse égyptienne ( ${ }^{\circ} 22,1 \mathrm{er}$ trimestre 1986) a consacré un dossier de presse aux élections soudanaises d'avril 19S6, sous le titre : « Des élections libres au Soudan » (pp. 55-133), précédé d'une introduction de F. IRETON : « Le contexte des élections » (pp. 1-54). Un autre dossier sur l'actualité soudanaise a été publié par la Revue de la presse égyptienne au 1er semestre 1989 (n³4, pp. 90-201), après les inondations de l'été 1988 et juste avant le coup d'État militaire du 30 juin 1989 ; ce dossier, composé et introduit par Habib Ayeb, était intitulé « Soudan ; géopolitique 
d'une crise ». De plus, à partir de son numéro 19 (2 $2^{\mathrm{e}}$ trimestre 1985) la Revue de la presse égyptienne a intégré le Soudan à sa « chronologie politique et sociale ». Dans le numéro 19 du Bulletin du CEDEJ (1er semestre 1986) est paru un dossier intitulé « Eléments pour une économie politique de la vallée du Nil », formant les actes d'une table ronde tenue sur ce thème au CEDEJ en décembre 1985 et comportant une douzaine d'articles, dont un certain nombre traitaient directement du Soudan (pp. 111-170) : Arif Jamal M. Ahmad, « Another aspect of irrigated agriculture in Sudan : the Northern Province »; Marc Lavergne, Frédéric Gheeraert, « La colonisation agricole dans la région de Damazîn (Province du Nil Bleu) »; Marc Lavergne, « La Guezira : les paysans et la production ». Enfin, dans le numéro 22 de la même publication ( $2^{\mathrm{e}}$ semestre 1987 ), a paru un autre dossier intitulé « Le Soudan et la vallée du Nil », composé de dix articles dont sept traitaient spécifiquement du Soudan; l'on relève successivement, des pages 69 à 193 : Medani Mohammed Ahmed, « The political economy of development in the Soudan » et «L'économie politique du développement agricole au Soudan »; Arif Jamal M. Ahmed, « Réflexion sur le développement socio-économique de l'agriculture soudanaise... »; Marc Lavergne, " Exode rural et croissance urbaine au Soudan » ; Hassan Ahmed Ibrahim, « Historique des relations égypto-soudanaises »; Robert 0. Collins, « Perspectives du Sud-Soudan : 1850-1956 » et Gérard Prunier, « Chronologie du conflit sud-soudanais (1983-1987)».

3. La plupart de ces derniers semblent en effet méconnaître la dimension moyenorientale rattachant le Soudan à un ensemble géopolitique dont d'autres tribulations focalisent l'attention et, sauf désastre dit « naturel » ou grandes manoeuvres internationales, ils n'ont Jamais prêté l'attention nécessaire, ni à la Corne de l'Afrique, ni à 1 »Afrique anglophone » dont ce pays fait également partie. Si ce dernier, lorsque sécheresse et famine n'y sévissent pas (mais il est bon d'en annoncer, alors même qu'elles ne sont pas en vue et d'en gonfler le chiffre des victimes probables) a parfois droit à quel qu'entrefilet ou à trente secondes de bulletin d'information, ce n'est guère, le plus souvent, que pour en stigmatiser la fonction de « nouvelle plate-forme du terrorisme islamiste international » et y révéler la présence d'un « Ayatollah de Khartoum » (à propos d'Hassan al-Tourabi, en couverture d'un hebdomadaire français d'informations générales, été 1993) quand ce n'est pas du « pape des musulmans » (en légende d'une photo du même dans Libération du 18/12/1993, p.18 ; « Hassan al-Tourabi rêve de devenir le pape des musulmans »). En ce qui concerne la presse, il y a lieu de mentionner une exception durable et notoire à ce noir tableau : il s'agit des articles du Monde Diplomatique traitant de l'actualité soudanaise, la plupart sous la plume d'Alain Gresh.

4. Articles prévus : Ch. Delmet (problèmes du Sud-Soudan), I. Dalmau (transformations économiques de l'élevage et de la commercialisation du cheptel camelin), D. Morin (changements linguistiques dans la région Est du Soudan), A. Tier (problèmes de l'application de la chiari'a), R. Hasterok (personnes déplacées), M.A.Ahmed (système éducatif soudanais), F. Burgat (mouvements islamistes), E. Conte et F. Ireton (évolution comparée des structures agraires et de la sécurité alimentaire dans le Nord-Kordofan et la province de Kassala).

5. Ce qui ne les empêche pas d'être très actifs, à preuve leur deux dernières publications collectives, qui constituent des références indispensables sur le Soudan : d'une part, sous la direction de Marc Lavergne, Le Soudan contemporain, Karthala/ CERMOC, Paris, 1989 (avec des contributions, pour ce qui est des chercheurs français, de G. Beaugé, H. Bleuchot, M. Chatelus, Ch. Delmet, D. Fawzi. N. Grandin, A. Gresh, M. 
Lavergne, R. Marchal, C. Miller et G. Prunier, auxquelles s'ajoutent celles de deux universitaires soudanais) et d'autre part, sous la direction de H. Bleuchot, Ch. Delmet et D. Hopwood, Sudan : History, Identity. Ideology / Histoire, identités, idéologies, lthaca Press, London. 1991 (avec des contributions de sept des onze chercheurs français ayant contribué au premier ouvrage cité, et de nombreux autres articles de chercheurs soudanais et européens). L'on trouve également, de manière régulière, des articles de sciences sociales concernant le Soudan dans les revues françaises suivantes : MaghrebMachrek, Politique Africaine, Cahiers d'Etudes Africaines, Islam et société au sud du Sahara. Les Cahiers de l'Orient, etc. A noter qu'il existe une Société française d'études soudanaises siégeant au Musée de l'Homme, à Paris, dont le responsable est Christian Delmet et qui organise régulièrement des conférences et envoie à ses membres, plusieurs lois par an, des bibliographies. Ces dernières sont l'occasion concrète de s'apercevoir que, si les publications en français sur le Soudan restent peu nombreuses, celles de langue anglaise sont en nombre considérable, tant au Soudan (beaucoup de " social scientist » soudanais écrivent en anglais) qu'en Grande-Bretagne, aux États-Unis, en Hollande (Institute of Social Studies, La Haye), dans les pays Scandinaves (Norvège en particulier, à l'Université de Bergen ; les grands ethnologues norvégiens que sont Friedrick Barth et Gunnar Sorbo ont travaillé ou travaillent au Soudan) et enfin en Allemagne (dont plusieurs universités ont des programmes de recherche sur le Soudan et où la revue Orient publie régulièrement des articles le concernant). De nombreux chercheurs soudanais publient dans ces pays, où ils ont souvent fait une partie de leurs études supérieures - doctorat en particulier.

INDEX

Mots-clés : Soudan

\section{AUTEURS}

ÉDOUARD CONTE

Cedej 